\title{
A METRIC DISCREPANCY RESULT WITH GIVEN SPEED
}

\author{
ISTVÁN BERKES, KATUSI FUKUYAMA, AND TAKUYA NISHIMURA
}

\begin{abstract}
It is known that the discrepancy $D_{N}\{k x\}$ of the sequence $\{k x\}$ satisfies $N D_{N}\{k x\}=O\left((\log N)(\log \log N)^{1+\varepsilon}\right)$ a.e. for all $\varepsilon>0$, but not for $\varepsilon=0$. For $n_{k}=\theta^{k}, \theta>1$ we have $N D_{N}\left\{n_{k} x\right\} \leq\left(\Sigma_{\theta}+\varepsilon\right)(2 N \log \log N)^{1 / 2}$ a.e. for some $0<\Sigma_{\theta}<\infty$ and $N \geq N_{0}$ if $\varepsilon>0$, but not for $\varepsilon<0$. In this paper we prove, extending results of Aistleitner-Larcher [6], that for any sufficiently smooth intermediate speed $\Psi(N)$ between $(\log N)(\log \log N)^{1+\varepsilon}$ and $(N \log \log N)^{1 / 2}$ and for any $\Sigma>0$, there exists a sequence $\left\{n_{k}\right\}$ of positive integers such that $N D_{N}\left\{n_{k} x\right\} \leq(\Sigma+\varepsilon) \Psi(N)$ eventually holds a.e. for $\varepsilon>0$, but not for $\varepsilon<0$. We also consider a similar problem on the growth of trigonometric sums.
\end{abstract}

\section{INTRODUCTION}

A sequence $\left\{x_{k}\right\}$ of real numbers is said to be uniformly distributed modulo 1 if

$$
\frac{1}{N} \#\left\{k \leq N:\left\langle x_{k}\right\rangle \in[a, b)\right\} \rightarrow b-a, \quad(N \rightarrow \infty),
$$

for all $0 \leq a<b \leq 1$, where $\langle x\rangle$ denotes the fractional part $x-$ $[x]$ of a real number $x$. The discrepancy $D_{N}\left\{x_{k}\right\}$, also denoted by $D_{N}\left(x_{1}, \ldots, x_{N}\right)$, is used to measure the speed of convergence:

$$
D_{N}\left\{x_{k}\right\}=\sup _{0 \leq a<b \leq 1}\left|\frac{1}{N} \#\left\{k \leq N:\left\langle x_{k}\right\rangle \in[a, b)\right\}-(b-a)\right| .
$$

For arithmetic progressions $\{k x\}$ with $x \notin \mathbf{Q}$, Bohl [10], Sierpiński [24], and Weyl [26] independently proved that they are uniformly distributed modulo 1. A metric result of Khintchine [20] implies

$$
N D_{N}\{k x\}=O\left((\log N)(\log \log N)^{1+\varepsilon}\right) \quad \text { a.e. for any } \varepsilon>0
$$

and this fails for $\varepsilon \leq 0$. The discrepancy of exponentially growing sequences has also been investigated extensively. By assuming the

I. Berkes is supported by FWF Grant P24302-N18 and OTKA Grant K108615. K. Fukuyama is supported by JSPS KAKENHI 16K05204. 
Hadamard gap condition

$$
n_{k+1} / n_{k} \geq q>1 \quad(k=1,2, \ldots),
$$

Philipp [23] proved, using Takahashi's method [25], that

$$
\frac{1}{4 \sqrt{2}} \leq \varlimsup_{N \rightarrow \infty} \frac{N D_{N}\left\{n_{k} x\right\}}{\sqrt{2 N \log \log N}} \leq \frac{1}{\sqrt{2}}\left(166+\frac{664}{q^{1 / 2}-1}\right) \text { a.e. }
$$

For improvements of (3), see [3] for the lower bound, and [18] for the upper bound. In case of geometric progressions, an exact law of the iterated logarithm holds: for any $\theta \notin[-1,1]$ there exists a constant $\Sigma_{\theta} \geq 1 / 2$ with

$$
\varlimsup_{N \rightarrow \infty} \frac{N D_{N}\left\{\theta^{k} x\right\}}{\sqrt{2 N \log \log N}}=\Sigma_{\theta} \quad \text { a.e. }
$$

If $\theta^{j} \notin \mathbf{Q}$ for any $j \in \mathbf{N}$, then $\Sigma_{\theta}=\frac{1}{2}$, otherwise $\Sigma_{\theta}>\frac{1}{2}$. For a $\theta$ which is a power root of an integer, of a large rational number, or of a ratio of odd integers, the concrete value of $\Sigma_{\theta}$ is evaluated. See $[12,14,15,16,17]$. For conditions to have an exact law of the iterated logarithm in (3), see $[1,5]$.

Since there is a big difference between (1) and (3), it is natural to ask if for intermediate speeds $\Psi(N)$ between $(\log N)(\log \log N)^{1+\varepsilon}$ and $(N \log \log N)^{1 / 2}$ one can find a sequence $\left\{n_{k}\right\}$ of integers such that the growth speed of $D_{N}\left\{n_{k} x\right\}$ is $\Psi(N)$ in the above sense. For all $\gamma \in$ $(0,1 / 2]$, Aistleitner and Larcher [6] constructed an increasing sequence $\left\{n_{k}\right\}$ of integers such that $N D_{N}\left\{n_{k} x\right\}=O\left(N^{\gamma}\right)$ and $N D_{N}\left\{n_{k} x\right\}=$ $\Omega\left(N^{\gamma-\varepsilon}\right)$ a.e. for all $\varepsilon>0$. They also constructed (see [7]) a sequence $\left\{n_{k}\right\}$ with polynomial growth such that $N D_{N}\left\{n_{k} x\right\}=O\left((\log N)^{2+\varepsilon}\right)$ a.e. for all $\varepsilon>0$.

The main result of the present paper is the following

Theorem 1. Let $\{\Psi(N)\}$ be a sequence of real numbers. Assume that there exists a constant $N_{0}$ such that

$$
\begin{gathered}
0<\Psi(N) \leq \Psi(N+1) \quad \text { for all } N \geq N_{0}, \\
\Psi(N) \geq(\log N)(\log \log N)^{1+\varepsilon} \quad \text { for some } \varepsilon>0 \text { and } N \geq N_{0}, \\
\Psi^{2}(N+1)-\Psi^{2}(N)=o\left(\log \log \Psi^{2}(N)\right) .
\end{gathered}
$$

Then for any $\Sigma>0$, there exists a sequence $\left\{n_{k}\right\}$ of positive integers satisfying $1 \leq n_{k+1}-n_{k} \leq 2$ and

$$
\varlimsup_{N \rightarrow \infty} \frac{N D_{N}\left\{n_{k} x\right\}}{\Psi(N)}=\Sigma \quad \text { a.e. }
$$


Note that for the function $\Psi^{2}(N)=N \log \log N$ we have

$$
\Psi^{2}(N+1)-\Psi^{2}(N) \sim \log \log \Psi^{2}(N)
$$

and thus condition (6) means that the jumps of $\Psi^{2}(N)$ are of smaller order of magnitude than those of $N \log \log N$. Naturally, this implies that $\Psi^{2}(N)=o(N \log \log N)$ and thus the conditions of Theorem 1 bound the function $\Psi^{2}(N)$ between $(\log N)(\log \log N)^{1+\varepsilon}$ and $N \log \log N$ and require a certain smoothness of growth. Typical examples are $\Psi(N)=$ $N^{\alpha}(\log N)^{\beta}(\log \log N)^{\gamma}$ where the parameters $\alpha, \beta, \gamma$ are chosen so that the order of growth of $\Psi^{2}(N)$ is between the previous bounds. Note that the theorem does not cover $\Psi(N)=(N \log \log N)^{1 / 2}$; the existence of $\left\{n_{k}\right\}$ with (7) is already proved in [4] for $0<\Sigma<\infty$, and in [2] for $\Sigma=\infty$. See also $[9,14]$.

As a related problem, we can ask if there exists a sequence $\left\{n_{k}\right\}$ such that $\sum_{k=1}^{N} \cos 2 \pi n_{k} x$ grows with a given speed $\Psi(N)$. The law of the iterated logarithm by Erdős-Gál [11] states

$$
\varlimsup_{N \rightarrow \infty} \frac{1}{\sqrt{N \log \log N}} \sum_{k=1}^{N} \cos 2 \pi n_{k} x=1 \quad \text { a.e. }
$$

for $\left\{n_{k}\right\}$ satisfying the Hadamard gap condition (2). As we will see in Section 4, for any $D>0$ there exists an increasing $\left\{n_{k}\right\}$ such that (8) holds with the norming factor replaced by $c \sqrt{N}(\log \log N)^{D}$. The following theorem shows that any growth speed $O\left(\sqrt{N}(\log \log N)^{D}\right)$ with small jumps is possible for $\sum_{k=1}^{N} \cos 2 \pi n_{k} x$.

Theorem 2. Let $\{\Psi(N)\}$ be an sequence of real numbers. Assume that there exists a constant $N_{0}$ and $D>0$ such that (4),

$$
\Psi(N) \rightarrow \infty, \quad \text { and } \quad \Psi^{2}(N+1)-\Psi^{2}(N)=o\left(\left(\log \log \Psi^{2}(N)\right)^{D}\right) .
$$

Then there exists a strictly increasing sequence $\left\{n_{k}\right\}$ of positive integers such that

$$
\varlimsup_{N \rightarrow \infty} \frac{1}{\Psi(N)} \sum_{k=1}^{N} \cos 2 \pi n_{k} x=1 \quad \text { a.e. }
$$

In conclusion, we mention a number of open problems related to our results. Let $\mathcal{G}$ denote the class of functions $\Psi(N), N=1,2, \ldots$ such that for some increasing sequence $\left\{n_{k}\right\}$ relation (7) holds for some constant $0<\Sigma<\infty$. From Theorem 1 it follows that $\mathcal{G}$ contains all smoothly increasing functions $\Psi(N)$ with speed between $(\log N)(\log \log N)^{1+\varepsilon}$ for some $\varepsilon>0$ and $(N \log \log N)^{1 / 2}$. By a classical result of W. Schmidt (see e.g. Kuipers and Niedereiter [22], p. 109) for any infinite sequence $\left\{x_{k}\right\}$ we have $N D_{N}\left\{x_{k}\right\} \geq c \log N$ for 
infinitely many $N$ with an absolute constant $c$ and thus $\mathcal{G}$ contains no functions $\Psi(N)=o(\log N)$. Hence assumption (5) in Theorem 1 is nearly optimal; whether $\Psi(N)=(\log N)(\log \log N)^{\alpha}, 0 \leq \alpha \leq 1$ belongs to $\mathcal{G}$ remains open. Concerning upper bounds for functions in $\mathcal{G}$, the results of Baker [8] and Berkes and Philipp [9] imply that

$$
N D_{N}\left\{n_{k} x\right\} \leq \text { const } \cdot N^{1 / 2}(\log N)^{\gamma} \text { a.e. }
$$

holds for all $\left\{n_{k}\right\}$ if $\gamma>3 / 2$ but not if $\gamma \leq 1 / 2$. This implies that for $\gamma>3 / 2$ we have $N^{1 / 2}(\log N)^{\gamma} \notin \mathcal{G}$ and makes it plausible (but does not prove) that $(N \log N)^{1 / 2} \in \mathcal{G}$. If this is true, condition (6) in Theorem 1 can be replaced by

$$
\Psi^{2}(N+1)-\Psi^{2}(N)=o\left(\log \Psi^{2}(N)\right)
$$

allowing all smoothly growing functions $\Psi(N)=O(N \log N)^{1 / 2}$, an essentially optimal result. Similar remarks hold for Theorem 2.

\section{Key Proposition}

We begin with proving a weaker version of Theorem 1 .

Proposition 3. For any sequence $\{\psi(N)\}$ satisfying

$$
\begin{gathered}
\psi(0)=0, \quad \psi(N) \leq \psi(N+1), \\
(\log N)(\log \log N)^{1+\varepsilon}=o(\psi(N)) \quad \text { for some } \quad \varepsilon>0, \\
\psi^{2}(N+1)-\psi^{2}(N) \leq \frac{1}{2}\left(4 \vee \log \log \psi^{2}(N)\right),
\end{gathered}
$$

there exists a sequence $\left\{n_{k}\right\}$ of positive integers satisfying $1 \leq n_{k+1}-$ $n_{k} \leq 2$ and

$$
\varlimsup_{N \rightarrow \infty} \frac{N D_{N}\left\{n_{k} x\right\}}{\psi(N)}=\frac{\sqrt{2}}{4} \quad \text { a.e. }
$$

Set $G(x)=x /(4 \vee \log \log x)$, where $\log \log x$ is meant as $-\infty$ for $x \leq 1$. Note that $G(x)$ is increasing. By (12), we can derive

$$
G\left(\psi^{2}(N+1)\right)-G\left(\psi^{2}(N)\right) \leq \frac{\psi^{2}(N+1)-\psi^{2}(N)}{4 \vee \log \log \psi^{2}(N)} \leq \frac{1}{2}
$$

Let $\nu_{i}$ be the smallest $\nu$ satisfying $2 i^{3} \leq G\left(\psi^{2}\left(i^{3}+\nu\right)\right)$. Note that $\nu_{0}=0$. By (14), we have

$$
G\left(\psi^{2}\left(i^{3}+\nu_{i}\right)\right)=2 i^{3}+e_{i} \text { for some } 0 \leq e_{i}<1 / 2 .
$$

Set $\Delta_{i}=\mathbf{N} \cap\left(2(i-1)^{3}, 2 i^{3}\right]$ and $\eta_{i}=2 i^{3}-2(i-1)^{3}$. 
By using (14), we have

$$
\begin{aligned}
& \eta_{i}-\frac{1}{2} \leq 2 i^{3}-2(i-1)^{3}+e_{i}-e_{i-1} \\
& =G\left(\psi^{2}\left(i^{3}+\nu_{i}\right)\right)-G\left(\psi^{2}\left((i-1)^{3}+\nu_{i-1}\right)\right) \leq \frac{1}{2}\left(\frac{1}{2} \eta_{i}+\nu_{i}-\nu_{i-1}\right) .
\end{aligned}
$$

By $\eta_{i} \geq 2$, we have

$$
\nu_{i}-\nu_{i-1} \geq(3 / 2) \eta_{i}-1 \geq \eta_{i} \quad \text { and } \quad \nu_{i} \geq 2 i^{3} .
$$

Set $\mu_{k}=2 \nu_{i}+2\left(k-2 i^{3}\right)$ for $k \in \Delta_{i}$. By $\mu_{2 i^{3}+1}=2 \nu_{i+1}-2 \eta_{i+1}+2 \geq$ $2 \nu_{i}+2>\mu_{2 i^{3}}$, we see that $\left\{\mu_{k}\right\}$ is strictly increasing.

We now introduce some notation. Denote by $\mathbf{1}_{[a, b)}$ the indicator function of $[a, b)$, and put $\widetilde{\mathbf{1}}_{[a, b)}\langle x\rangle=\mathbf{1}_{[a, b)}(\langle x\rangle)-(b-a)$. Then we have

$$
N D_{N}\left\{x_{k}\right\}=N D_{N}\left(x_{1}, \ldots, x_{N}\right)=\sup _{0 \leq a<b \leq 1}\left|\sum_{k=1}^{N} \widetilde{\mathbf{1}}_{[a, b)}\left\langle x_{k}\right\rangle\right| .
$$

Put $S=\left\{2^{-l} i: l \in \mathbf{N}, i=0,1, \ldots, 2^{l}\right\}, S^{2<}=\{(a, b): a, b \in S, a<b\}$, $\phi_{C}(t)=\sqrt{C t(1 \vee \log \log t)}$, and $\sigma_{a, b}=\sqrt{(b-a)(1-(b-a))}$. Let $\left\{X_{k}\right\}$ be a sequence of independent random variables satisfying $P\left(X_{k}=\right.$ 1) $=P\left(X_{k}=-1\right)=1 / 2$.

Lemma 4. We have

$$
\varlimsup_{N \rightarrow \infty} \frac{1}{\phi_{2}(N)}\left|\sum_{k=1}^{N} \widetilde{\mathbf{1}}_{[a, b)}\left\langle\mu_{k} x\right\rangle X_{k}\right|=\sigma_{a, b}
$$

for all $(a, b) \in S^{2<}$, a.e., a.s.

Proof. Since $\mu_{k}$ is a strictly increasing sequence of integers, by Weyl's theorem [27], $\left\{\mu_{k} x\right\}$ is uniformly distributed modulo 1 a.e. Hence,

$$
B_{N}:=\sum_{k=1}^{N} \widetilde{\mathbf{1}}_{[a, b)}^{2}\left\langle\mu_{k} x\right\rangle \sim N \int_{0}^{1} \widetilde{\mathbf{1}}_{[a, b)}^{2}(y) d y=N \sigma_{a, b}^{2} \rightarrow \infty \quad \text { a.e. }
$$

if $b-a \neq 0,1$. By Kolmogorov's law of the iterated logarithm [21]

$$
\varlimsup_{N \rightarrow \infty} \frac{1}{\phi_{2}\left(B_{N}\right)}\left|\sum_{k=1}^{N} \widetilde{\mathbf{1}}_{[a, b)}\left\langle\mu_{k} x\right\rangle X_{k}\right|=1 \quad \text { a.s., a.e., }
$$

we see that (17) holds a.s., a.e. if $0<b-a<1$. Clearly (17) holds if $b-a=0,1$. Since $S^{2<}$ is countable, we see that (17) holds for all $(a, b) \in S^{2<}$, a.s., a.e. By Fubini's theorem, we have the conclusion.

Lemma 5. Suppose that $l \in \mathbf{N}$ and $0 \leq i<2^{l}$, we have

$$
\varlimsup_{N \rightarrow \infty} \frac{1}{\phi_{2}(N)} \sup _{0<c<2^{-l}}\left|\sum_{k=1}^{N} \widetilde{\mathbf{1}}_{\left[2^{-l} i, 2^{-l} i+c\right)}\left\langle\mu_{k} x\right\rangle X_{k}\right| \leq 4 \cdot 2^{-l / 2} \quad \text { a.e., a.s. }
$$


Proof. Denote $\mathbf{1}_{[a, b)}(\langle x\rangle)$ simply by $\mathbf{1}_{[a, b)}\langle x\rangle$. By noting

$$
b_{N}=\sum_{k=1}^{N} \mathbf{1}_{\left[2^{-l} i, 2^{-l}(i+1)\right)}\left\langle\mu_{k} x\right\rangle \sim N \int_{0}^{1} \mathbf{1}_{\left[2^{\left.-l i, 2^{-l}(i+1)\right)}\right.}(y) d y=N 2^{-l} \quad \text { a.e. }
$$

and by following the proof of Lemma 4 of [13], we can prove

$$
\varlimsup_{N \rightarrow \infty} \frac{1}{\phi_{2}(N)} \sup _{0<c<2^{-l}}\left|\sum_{k=1}^{N} \mathbf{1}_{\left[2^{-l} i, 2^{-l} i+c\right)}\left\langle\mu_{k} x\right\rangle X_{k}\right| \leq \sqrt{10 \cdot 2^{-l}} \quad \text { a.e., a.s. }
$$

Thus together with the law of the iterated logarithm

$$
\varlimsup_{N \rightarrow \infty} \sup _{0<c<2^{-l}} \frac{c}{\phi_{2}(N)}\left|\sum_{k=1}^{N} X_{k}\right|=\varlimsup_{N \rightarrow \infty} \frac{2^{-l}}{\phi_{2}(N)}\left|\sum_{k=1}^{N} X_{k}\right| \leq 2^{-l} \quad \text { a.s. },
$$

we have the conclusion.

For $0 \leq a<b \leq 1$, take $l$ with $b-a>2^{-l}$ and take the largest $i$ and $j$ such that $2^{-l} i \leq a<2^{-l} j \leq b$. Then we have $\mathbf{1}_{[a, b)}=\mathbf{1}_{\left[2^{-l} i, 2^{-l} j\right)}-$ $\mathbf{1}_{\left[2^{-l} i, a\right)}+\mathbf{1}_{\left[2^{-l} j, b\right)}$ and $\widetilde{\mathbf{1}}_{[a, b)}=\widetilde{\mathbf{1}}_{\left[2^{-l} i, 2^{-l} j\right)}-\widetilde{\mathbf{1}}_{\left[2^{-l} i, a\right)}+\widetilde{\mathbf{1}}_{\left[2^{-l} j, b\right)}$, which implies

$$
\begin{aligned}
& \max _{0 \leq i<j \leq 2^{l}} \varlimsup_{N \rightarrow \infty} \frac{1}{\phi_{2}(N)}\left|\sum_{k=1}^{N} \widetilde{\mathbf{1}}_{\left[2^{-l} i, 2^{-l} j\right)}\left\langle\mu_{k} x\right\rangle X_{k}\right| \\
& \leq \varlimsup_{N \rightarrow \infty} \sup _{0<a<b \leq 1} \frac{1}{\phi_{2}(N)}\left|\sum_{k=1}^{N} \widetilde{\mathbf{1}}_{[a, b)}\left\langle\mu_{k} x\right\rangle X_{k}\right| \\
& \leq \max _{0 \leq i<j \leq 2^{l}} \varlimsup_{N \rightarrow \infty} \frac{1}{\phi_{2}(N)}\left|\sum_{k=1}^{N} \widetilde{\mathbf{1}}_{\left[2^{-l} i, 2^{-l} j\right)}\left\langle\mu_{k} x\right\rangle X_{k}\right| \\
& \quad+2 \max _{0 \leq i \leq 2^{l}} \varlimsup_{N \rightarrow \infty} \sup _{0<a \leq 2^{-l}} \frac{1}{\phi_{2}(N)}\left|\sum_{k=1}^{N} \widetilde{\mathbf{1}}_{\left[2^{-l} i, 2^{-l} i+a\right)}\left\langle\mu_{k} x\right\rangle X_{k}\right| .
\end{aligned}
$$

By applying two lemmas above, we have

$$
\frac{1}{2} \leq \varlimsup_{N \rightarrow \infty} \sup _{0 \leq a<b \leq 1} \frac{1}{\phi_{2}(N)}\left|\sum_{k=1}^{N} \widetilde{\mathbf{1}}_{[a, b)}\left\langle\mu_{k} x\right\rangle X_{k}\right| \leq \frac{1}{2}+8 \cdot 2^{-l / 2} \quad \text { a.e., a.s. }
$$

which implies

$$
\varlimsup_{N \rightarrow \infty} \sup _{0 \leq a<b \leq 1} \frac{1}{\phi_{2}(N)}\left|\sum_{k=1}^{N} \widetilde{\mathbf{1}}_{[a, b)}\left\langle\mu_{k} x\right\rangle X_{k}\right|=\frac{1}{2} \quad \text { a.e., a.s. }
$$


By the relation $N D_{N}\left\{x_{k}+y\right\}=N D_{N}\left\{x_{k}\right\}$ and (1), we have $\eta_{i} D_{\eta_{i}}\left(\mu_{2(i-1)^{3}+1} x, \mu_{2(i-1)^{3}+2} x, \ldots, \mu_{2 i^{3}} x\right)=\eta_{i} D_{\eta_{i}}\{2 k x\}=O\left(\left(\log \eta_{i}\right)^{2}\right)$.

Noting $N D_{N}\left\{\mu_{k} x\right\} \leq \sum_{i=1}^{j} \eta_{i} D_{\eta_{i}}\left(\mu_{2(i-1)^{3}+1} x, \mu_{2(i-1)^{3}+2} x, \ldots, \mu_{2 i^{3}} x\right)$ for $N \in \Delta_{j}$, we have

$$
N D_{N}\left\{\mu_{k} x\right\}=O\left(\sum_{i=1}^{j}\left(\log \eta_{i}\right)^{2}\right)=O\left(N^{1 / 3}(\log N)^{2}\right)=o(\sqrt{N}) \quad \text { a.e. }
$$

by $j-1<(N / 2)^{1 / 3}$. This together with (18) implies

$$
\varlimsup_{N \rightarrow \infty} \sup _{0 \leq a<b \leq 1} \frac{1}{\phi_{2}(N)}\left|\sum_{k=1}^{N} \widetilde{\mathbf{1}}_{[a, b)}\left\langle\mu_{k} x\right\rangle \frac{X_{k}+1}{2}\right|=\frac{1}{4} \quad \text { a.e., a.s. }
$$

Note that $\left\{\mu_{k}\right\}$ and $\{2 k-1\}$ are mutually disjoint. Let $\left\{\lambda_{k}\right\}$ be an arrangement in increasing order of $\left\{\mu_{k}\right\} \cup\{2 k-1\}$. By $\mu_{2 i^{3}}=2 \nu_{i}$, we have $\#\left\{k: \mu_{k} \leq 2 \nu_{i}\right\}=2 i^{3}$ and $\#\left\{k: 2 k-1 \leq 2 \nu_{i}\right\}=\nu_{i}$, and thereby we have $\#\left\{k: \lambda_{k} \leq 2 \nu_{i}\right\}=2 i^{3}+\nu_{i}$ and $\lambda_{2 i^{3}+\nu_{i}}=2 \nu_{i}$. We set

$$
Y_{k}= \begin{cases}1 & \lambda_{k} \notin 2 \mathbf{N} \\ \left(X_{k}+1\right) / 2 & \lambda_{k} \in 2 \mathbf{N}\end{cases}
$$

$I_{N}=\#\left\{k \leq N: \lambda_{k} \notin 2 \mathbf{N}\right\}, J_{N}=\#\left\{k \leq N: Y_{k}=1, \lambda_{k} \in 2 \mathbf{N}\right\}$, and $H_{N}=\#\left\{k \leq N: Y_{k}=1\right\}=I_{N}+J_{N}$. We have $I_{2 i^{3}+\nu_{i}}=\#\{k \leq$ $\left.2 i^{3}+\nu_{i}: \lambda_{k} \notin 2 \mathbf{N}\right\}=\#\left\{k: 2 k-1 \leq 2 \nu_{i}\right\}=\nu_{i}$ and $H_{2 i^{3}+\nu_{i}}=J_{2 i^{3}+\nu_{i}}+\nu_{i}$. By the law of large numbers we have $J_{2 i^{3}+\nu_{i}} \sim \frac{1}{2} \#\left\{k: \mu_{k} \leq 2 \nu_{i}\right\}=i^{3}$ a.s. By (14), we have

$$
\left|G\left(\psi^{2}\left(H_{2 i^{3}+\nu_{i}}\right)\right)-G\left(\psi^{2}\left(i^{3}+\nu_{i}\right)\right)\right| \leq \frac{1}{2}\left|H_{2 i^{3}+\nu_{i}}-\left(i^{3}+\nu_{i}\right)\right|=\frac{1}{2}\left|J_{2 i^{3}+\nu_{i}}-i^{3}\right| .
$$

Dividing by $G\left(\psi^{2}\left(i^{3}+\nu_{i}\right)\right)=2 i^{2}+e_{i}$, we have

$$
\left|\frac{G\left(\psi^{2}\left(H_{2 i^{3}+\nu_{i}}\right)\right)}{2 i^{3}+e_{i}}-1\right| \leq \frac{1}{2}\left|\frac{J_{2 i^{3}+\nu_{i}}}{2 i^{3}+e_{i}}-\frac{i^{3}}{2 i^{3}+e_{i}}\right| \rightarrow 0 \quad \text { a.s. }
$$

Therefore we have $G\left(\psi^{2}\left(H_{2 i^{3}+\nu_{i}}\right)\right) \sim 2 i^{3}+e_{i} \sim 2 i^{3} \sim 2 J_{2 i^{3}+\nu_{i}}$ a.s. Since $J_{N}$ and $H_{N}$ are increasing, for $N \in\left[(i-1)^{3}+\nu_{i-1}, i^{3}+\nu_{i}\right]$ we have

$$
1 \sim \frac{G\left(\psi^{2}\left(H_{2(i-1)^{3}+\nu_{i-1}}\right)\right)}{2 J_{2 i^{3}+\nu_{i}}} \leq \frac{G\left(\psi^{2}\left(H_{N}\right)\right)}{2 J_{N}} \leq \frac{G\left(\psi^{2}\left(H_{2 i^{3}+\nu_{i}}\right)\right)}{2 J_{2(i-1)^{3}+\nu_{i-1}}} \sim 1
$$

and thereby,

$$
2 J_{N} \sim G\left(\psi^{2}\left(H_{N}\right)\right) \quad \text { a.s. }
$$


By (1), we see $N D_{N}\{(2 k-1) x\}=O\left((\log N)(\log \log N)^{1+\varepsilon / 2}\right)$, which implies $N D_{N}\{(2 k-1) x\}=o\left((\log N)(\log \log N)^{1+\varepsilon}\right)$ or

$$
\lim _{N \rightarrow \infty} \sup _{0 \leq a<b \leq 1} \frac{1}{A_{N}}\left|\sum_{k \leq N: \lambda_{k} \notin 2 \mathbf{N}} \widetilde{\mathbf{1}}_{[a, b)}\left\langle\lambda_{k} x\right\rangle Y_{k}\right|=0 \quad \text { a.e., a.s. }
$$

for $A_{N}=\left(\log I_{N}\right)\left(\log \log I_{N}\right)^{\varepsilon}$. Since $H_{N} \geq I_{N}$, it is valid for $A_{N}=$ $\left(\log H_{N}\right)\left(\log \log H_{N}\right)^{\varepsilon}$. Because of $(11)$, we see that $(21)$ holds for $A_{N}=$ $\sqrt{2} \psi\left(H_{N}\right)$.

By (19), we have

$$
\varlimsup_{N \rightarrow \infty} \sup _{0 \leq a<b \leq 1} \frac{1}{A_{N}}\left|\sum_{k \leq N: \lambda_{k} \in 2 \mathbf{N}} \widetilde{\mathbf{1}}_{[a, b)}\left\langle\lambda_{k} x\right\rangle Y_{k}\right|=\frac{1}{4} \quad \text { a.e., a.s. }
$$

for $A_{N}=\phi_{2}\left(\#\left\{k \leq N: \lambda_{k} \in 2 \mathbf{N}\right\}\right)$. By $J_{N} \sim \frac{1}{2} \#\left\{k \leq N: \lambda_{k} \in 2 \mathbf{N}\right\}$ a.s., we see that $(22)$ is valid for $A_{N}=\sqrt{2} \phi_{2}\left(J_{N}\right) \sim \phi_{2}\left(2 J_{N}\right)$. (20) and $\phi_{2}^{2}\left(G\left(\psi^{2}(N)\right)\right) \sim 2 \psi^{2}(N)$ imply $\phi_{2}^{2}\left(J_{N}\right) \sim \phi_{2}^{2}\left(G\left(\psi^{2}\left(H_{N}\right)\right)\right) / 2 \sim \psi^{2}\left(H_{N}\right)$ a.s. Hence $(22)$ holds for $A_{N}=\sqrt{2} \psi\left(H_{N}\right)$. Combining these, we have

$$
\varlimsup_{N \rightarrow \infty} \sup _{0 \leq a<b \leq 1} \frac{1}{\sqrt{2} \psi\left(H_{N}\right)}\left|\sum_{k=1}^{N} \widetilde{\mathbf{1}}_{[a, b)}\left\langle\lambda_{k} x\right\rangle Y_{k}\right|=\frac{1}{4} \quad \text { a.e., a.s. }
$$

Denoting by $\left\{n_{k}\right\}$ the subsequence $\left\{\lambda_{k}: Y_{k}=1\right\}$, we have (13) a.s.

\section{Proof of Theorem 1}

By (6), we have $\Psi^{2}(N)=o\left(N \log \log \Psi^{2}(N)\right)$ and $G\left(\Psi^{2}(N)\right)=o(N)$. For any $C>0$, we see $G\left(\phi_{C}^{2}(N)\right) \sim C N$ and hence $G\left(\Psi^{2}(N)\right) \leq$ $G\left(\phi_{C}^{2}(N)\right)$ or $\Psi^{2}(N) \leq \phi_{C}^{2}(N)$ for large $N$. Since it holds for any $C>0$, we see that $\Psi^{2}(N)=o\left(\phi_{C}^{2}(N)\right)$.

By (6), we can take $N_{1}>N_{0}$ such that for all $N \geq N_{1}$,

$$
(2 \sqrt{2} \Sigma \Psi(N+1))^{2}-(2 \sqrt{2} \Sigma \Psi(N))^{2} \leq \frac{1}{2} \log \log (2 \sqrt{2} \Sigma \Psi(N))^{2} .
$$

Take $c \in\left(0, \frac{1}{4}\right)$ such that $\phi_{c}^{2}\left(N_{1}\right)<\left(2 \sqrt{2} \Sigma \Psi\left(N_{1}\right)\right)^{2}$ holds. We have $(2 \sqrt{2} \Sigma \Psi(N))^{2}<\phi_{c}^{2}(N)$ for large $N \geq N_{1}$. Denote $N_{2}$ the minimum of such $N$. Putting

$$
\psi(N)= \begin{cases}\phi_{c}(N) & N<N_{2} \\ 2 \sqrt{2} \Sigma \Psi(N) & N \geq N_{2}\end{cases}
$$

it is clear that $\psi(N)$ satisfies (10) and (11). As to the condition (12), we first prove it for $\phi_{c}^{2}(N)$.

In the case $\log \log (N+1) \geq 1$, i.e. $N \geq 15$, we see $(N+1)(\log \log (N+$ 1) $-\log \log N) \leq((N+1) / N) / \log N \leq 2 / \log 15<\log \log 15 \leq$ 
$\log \log N$ and $(N+1) \log \log (N+1)-N \log \log N<2 \log \log N$. If $c \log \log N \leq 1$, then $2 c \log \log N \leq 2 \leq \frac{1}{2}\left(4 \vee \log \log \phi_{c}^{2}(N)\right)$. If $c \log \log N \geq 1$, then $2 c \log \log N \leq \frac{1}{2} \log \log N \leq \frac{1}{2} \log \log (c N \log \log N) \leq$ $\frac{1}{2}\left(4 \vee \log \log \phi_{c}^{2}(N)\right)$. Therefore, when $\log \log (N+1) \geq 1$, we have $\phi_{c}^{2}(N+1)-\phi_{c}^{2}(N) \leq 2 c \log \log N \leq \frac{1}{2}\left(4 \vee \log \log \phi_{c}^{2}(N)\right)$. When $\log \log (N+1) \leq 1$, clearly we have $\phi_{c}^{2}(N+1)-\phi_{c}^{2}(N) \leq c \leq \frac{1}{4} \leq$ $\frac{1}{2}\left(4 \vee \log \log \phi_{c}^{2}(N)\right)$.

By $\psi^{2}\left(N_{2}\right)-\psi^{2}\left(N_{2}-1\right) \leq\left(2 \sqrt{2} \Sigma \Psi\left(N_{2}\right)\right)^{2}-\phi_{c}^{2}\left(N_{2}-1\right) \leq \phi_{c}^{2}\left(N_{2}\right)-$ $\phi_{c}^{2}\left(N_{2}-1\right)$ together with $(23)$, we conclude that $\psi(N)$ satisfies $(12)$.

Hence we can apply Proposition 3 to have the conclusion.

\section{Proof of Theorem 2}

Take an integer $d \geq D \vee 2$ to satisfy

$$
\Psi^{2}(N+1)-\Psi^{2}(N)=o\left(\left(\log \log \Psi^{2}(N)\right)^{d}\right) .
$$

Put $M_{k}=2^{d-1}\left(\begin{array}{l}k \\ d\end{array}\right), L_{k}=\min \left\{n \mid \Psi^{2}(n) \geq\left(2^{d-1} / d !\right) M_{k}\left(\log \log M_{k}\right)^{d}\right\}$, and $L_{k}^{+}=L_{k}+M_{k+1}-M_{k}$.

There exists $K_{-}$such that $\max _{N \leq N_{0}} \Psi(N)<\left(2^{d-1} / d !\right) M_{k}\left(\log \log M_{k}\right)^{d}$ for all $k \geq K_{-}$. From now on, we consider only for $k \geq K_{-}$, for which we have $L_{k}>N_{0}$.

By $(24)$ and $\Psi^{2}\left(L_{k}-1\right)<\left(2^{d-1} / d !\right) M_{k}\left(\log \log M_{k}\right)^{d}$, we have

$$
\begin{aligned}
& \left(2^{d-1} / d !\right) M_{k}\left(\log \log M_{k}\right)^{d} \leq \Psi^{2}\left(L_{k}\right) \\
& =o\left(\left(\log \log \Psi^{2}\left(L_{k}-1\right)\right)^{d}\right)+\Psi^{2}\left(L_{k}-1\right) \\
& \leq o\left(\left(\log \log \left(M_{k}\left(\log \log M_{k}\right)^{d}\right)\right)+\left(2^{d-1} / d !\right) M_{k}\left(\log \log M_{k}\right)^{d},\right.
\end{aligned}
$$

$\Psi^{2}\left(L_{k}\right) /\left(2^{d-1} / d !\right) M_{k}\left(\log \log M_{k}\right)^{d} \rightarrow 1, \log \log \Psi^{2}\left(L_{k}\right)-\log \log M_{k} \rightarrow 0$ and $\log \log \Psi^{2}\left(L_{k}\right) \sim \log \log M_{k}$ in turn. Combining

$$
\begin{aligned}
& \Psi^{2}\left(L_{k+1}\right)-\Psi^{2}\left(L_{k}-1\right) \\
& \geq\left(2^{d-1} / d !\right)\left(M_{k+1}\left(\log \log M_{k+1}\right)^{d}-M_{k}\left(\log \log M_{k}\right)^{d}\right) \\
& \geq\left(2^{d-1} / d !\right)\left(M_{k+1}-M_{k}\right)\left(\log \log M_{k+1}\right)^{d}
\end{aligned}
$$

and $\Psi^{2}\left(L_{k+1}\right)-\Psi^{2}\left(L_{k}-1\right)=\left(L_{k+1}-L_{k}+1\right) o\left(\left(\log \log \Psi^{2}\left(L_{k+1}\right)\right)^{d}\right)$, we have

$$
\frac{M_{k+1}-M_{k}}{L_{k+1}-L_{k}+1} \leq \frac{o\left(\left(\log \log \Psi^{2}\left(L_{k+1}\right)\right)^{d}\right)}{\left(2^{d-1} / d !\right)\left(\log \log M_{k+1}\right)^{d}}=o(1) .
$$

Hence we see that there exists a $K_{0}$ such that

$$
L_{k+1}-L_{k}>M_{k+1}-M_{k} \text { i.e., } \quad L_{k+1}>L_{k}^{+} \quad\left(k \geq K_{0}\right) .
$$


By (24) we have $\Psi^{2}(N) \leq o\left(N\left(\log \log \Psi^{2}(N)\right)^{d}\right)$, Thereby $\log \Psi^{2}(N)<$ $\log N+d \log \log \log \Psi^{2}(N)$, and $\log \Psi^{2}(N) \leq 2 \log N$ or $\Psi^{2}(N) \leq N^{2}$ for large $\mathrm{N}$. Hence $\Psi^{2}(N)=o\left(N(\log \log N)^{d}\right)$. Hence we see $\Psi^{2}\left(M_{k}\right)=$ $o\left(M_{k}\left(\log \log M_{k}\right)^{d}\right)=o\left(\Psi^{2}\left(L_{k}\right)\right)$. It implies $M_{k}<L_{k}$ for large $k$. Take such $k \geq K_{0}$ and denote by $k_{0}$. We see $M_{k_{0}}<L_{k_{0}}$.

We define an non-decreasing sequence $\left\{a_{k}\right\}$ of positive integers as below. Put $a_{1}=\cdots=a_{k_{0}}=3$, take $a_{k_{0}+1}$ large enough to satisfy $a_{k_{0}+1} \geq a_{k_{0}}$ and

$$
\gamma_{k_{0}+1}^{+}:=\frac{1}{2} a_{k_{0}+1}^{k_{0}+1} \geq \frac{3}{2} a_{k_{0}}^{k_{0}}+\left(L_{k_{0}}-1-M_{k_{0}}\right)=: \gamma_{k_{0}+1}^{-}
$$

For $k \geq k_{0}$, inductively take $a_{k+2}$ large enough to satisfy $a_{k+2} \geq a_{k+1}$ and

$$
\gamma_{k+2}^{+}:=\frac{1}{2} a_{k+2}^{k+2} \geq \frac{3}{2} a_{k+1}^{k+1}+\left(L_{k+1}-L_{k}^{+}\right)=: \gamma_{k+2}^{-}
$$

Put $\rho_{j}=a_{j}^{j}$. Since $\rho_{j}$ satisfies the Hadamard gap condition $\rho_{j+1} / \rho_{j} \geq$ $a_{j+1} \geq 3$, by the law of the iterated logarithm we have

$$
\varlimsup_{N \rightarrow \infty} \frac{1}{\phi_{1}(N)} \sum_{j=1}^{N} \cos 2 \pi \rho_{j} x=\varlimsup_{N \rightarrow \infty} \frac{1}{\phi_{1}(N)}\left|\sum_{j=1}^{N} \cos 2 \pi \rho_{j} x\right|=1 \quad \text { a.e. }
$$

From this, we drive

$$
\varlimsup_{N \rightarrow \infty} \frac{d !}{\phi_{1}(N)^{d}} \sum_{1 \leq m_{1}<\cdots<m_{d} \leq N} \prod_{j=1}^{d} \cos 2 \pi \rho_{m_{j}} x=1 \quad \text { a.e. }
$$

For a function $f\left(m_{1}, \ldots, m_{d}\right)$ on $\{1, \ldots, N\}^{d}$, define a signed measure $\nu$ on $\{1, \ldots, N\}^{d}$ by

$$
\nu(A)=\sum_{\left(m_{1}, \ldots, m_{d}\right) \in A} f\left(m_{1}, \ldots, m_{d}\right) \quad\left(A \subset\{1, \ldots, N\}^{d}\right) .
$$

Let $J=\{(j, k) \mid 1 \leq j, k \leq N, j \neq k\}$. For $(j, k) \in J$, put $A_{(j, k)}=$ $\left\{\left(m_{1}, \ldots, m_{d}\right) \in\{1, \ldots, N\}^{d} \mid m_{j}=m_{k}\right\}$.

Putting

$$
f\left(m_{1}, \ldots, m_{d}\right)=\prod_{j=1}^{d} \cos 2 \pi \rho_{m_{j}} x
$$


and by applying the inclusion-exclusion principle

$$
\begin{aligned}
\nu\left(\{1, \ldots, N\}^{d} \backslash \bigcup_{\boldsymbol{j} \in J} A_{\boldsymbol{j}}\right) & =\nu\left(\{1, \ldots, N\}^{d}\right)-\sum_{\boldsymbol{j} \in J} \nu\left(A_{\boldsymbol{j}}\right) \\
& +\sum_{\boldsymbol{j}_{1}, \boldsymbol{j}_{2} \in J: \boldsymbol{j}_{1} \neq \boldsymbol{j}_{2}} \nu\left(A_{\boldsymbol{j}_{1}} \cap A_{\boldsymbol{j}_{2}}\right)-\cdots+\nu\left(\bigcap_{\boldsymbol{j} \in J} A_{\boldsymbol{j}}\right),
\end{aligned}
$$

we see that

$$
\left|\sum_{m_{1}, \ldots, m_{d} \leq N: m_{j} \neq m_{k}((j, k) \in J)} \prod_{j=1}^{d} \cos 2 \pi \rho_{m_{j}} x-\left(\sum_{k=1}^{N} \cos 2 \pi \rho_{k} x\right)^{d}\right|
$$

can be bounded by a linear combination of

$$
\left|\prod_{j=1}^{\beta} \sum_{k=1}^{N} \cos ^{\alpha_{j}} 2 \pi \rho_{k} x\right| \quad\left(\alpha_{1}+\cdots+\alpha_{\beta}=d, \max _{j=1}^{\beta} \alpha_{j} \geq 2\right) .
$$

Note that we can verify

$$
\begin{aligned}
0 & \leq \varlimsup_{N \rightarrow \infty} \frac{1}{\phi_{1}(N)^{d}}\left|\prod_{j=1}^{\beta} \sum_{k=1}^{N} \cos ^{\alpha_{j}} 2 \pi \rho_{k} x\right| \\
& \leq \prod_{j=1}^{\beta} \varlimsup_{N \rightarrow \infty} \frac{1}{\phi_{1}(N)^{\alpha_{j}}}\left|\sum_{k=1}^{N} \cos ^{\alpha_{j}} 2 \pi \rho_{k} x\right|=0 \quad \text { a.e. }
\end{aligned}
$$

because

$$
\varlimsup_{N \rightarrow \infty} \frac{1}{\phi_{1}(N)^{\alpha}}\left|\sum_{k=1}^{N} \cos ^{\alpha} 2 \pi \rho_{k} x\right| \leq \varlimsup_{N \rightarrow \infty} \frac{N}{\phi_{1}(N)^{\alpha}}=0
$$

holds for $\alpha \geq 2$. Hence by (28) we have

$$
\begin{gathered}
\varlimsup_{N \rightarrow \infty} \frac{1}{\phi_{1}(N)^{d}} \sum_{m_{1}, \ldots, m_{d} \leq N: m_{j} \neq m_{k}((j, k) \in J)} \prod_{j=1}^{d} \cos 2 \pi \rho_{m_{j}} x \\
\quad=\varlimsup_{N \rightarrow \infty} \frac{1}{\phi_{1}(N)^{d}}\left(\sum_{k=1}^{N} \cos 2 \pi \rho_{k} x\right)^{d}=1 \quad \text { a.e. }
\end{gathered}
$$

and thereby we see (29).

Let $\mathcal{S}_{0}$ be a collection of $\left(b_{1}, b_{2}, \ldots\right) \in\{-1,0,1\}^{\mathbf{N}}$ such that $b_{i}=0$ for all large $i$.

Lemma 6. The mapping $\mathcal{S}_{0} \ni\left(b_{1}, b_{2}, \ldots\right) \mapsto \sum_{i=1}^{\infty} b_{i} a_{i}^{i} \in \mathbf{Z}$ is injective. 
Proof. Because of $\left|\sum_{i=1}^{I-1} b_{i} a_{i}^{i}\right| \leq \sum_{i=1}^{I-1} a_{I-1}^{i}<\frac{1}{2} a_{I}^{I}$, we have

$$
\sum_{i=1}^{I} b_{i} a_{i}^{i} \in\left(\left(b_{I}-\frac{1}{2}\right) a_{I}^{I},\left(b_{I}+\frac{1}{2}\right) a_{I}^{I}\right)
$$

and if $b_{I} \neq 0$, then

$$
\sum_{i=1}^{I} b_{i} a_{i}^{i} \in\left(-\frac{3}{2} a_{I}^{I},-\frac{1}{2} a_{I}^{I}\right) \cup\left(\frac{1}{2} a_{I}^{I}, \frac{3}{2} a_{I}^{I}\right)=: C_{I} .
$$

Take $\left(b_{1}, b_{2}, \ldots\right) \in \mathcal{S}_{0}$ and $\left(b_{1}^{\prime}, b_{2}^{\prime}, \ldots\right) \in \mathcal{S}_{0}$ and assume $\sum_{i=1}^{\infty} b_{i} a_{i}^{i}=$ $\sum_{i=1}^{\infty} b_{i}^{\prime} a_{i}^{i}$. By putting $I=\max \left\{i \mid b_{i} \neq 0\right\}$ and $I^{\prime}=\max \left\{i \mid b_{i}^{\prime} \neq 0\right\}$, then we see that $\sum_{i=1}^{\infty} b_{i} a_{i}^{i} \in C_{I}$ and $\sum_{i=1}^{\infty} b_{i} a_{i}^{i} \in C_{I^{\prime}}$. By $\frac{3}{2} a_{I}^{I} \leq \frac{1}{2} a_{I+1}^{I+1}$, we see that $C_{I}(I=1,2, \ldots)$ are mutually disjoint and $\max \{i \mid$ $\left.b_{i} \neq 0\right\}=\max \left\{i \mid b_{i}^{\prime} \neq 0\right\}$. Because $\left(\left(b-\frac{1}{2}\right) a_{I}^{I},\left(b+\frac{1}{2}\right) a_{I}^{I}\right)(b \in \mathbf{Z})$ are mutually disjoint, we see $b_{I}=b_{I}^{\prime}$. Hence we have $\sum_{i=1}^{I-1} b_{i} a_{i}^{i}=$ $\sum_{i=1}^{I-1} b_{i}^{\prime} a_{i}^{i}$. In the same way, we can verify $b_{i}=b_{i}^{\prime}$ for all $i<I$, and see that the mapping is injective.

By this lemma, we see that

$$
\rho_{m_{d}}+\varepsilon_{d-1} \rho_{m_{d-1}}+\cdots+\varepsilon_{1} \rho_{m_{1}}
$$

with $m_{1}<m_{2}<\cdots<m_{d}$ and $\varepsilon_{1}, \ldots, \varepsilon_{d}= \pm 1$ are all distinct. Denote by $\left\{l_{i}\right\}$ the arrangement in increasing order of this family.

Note that $M_{k}$ equals to the number of the sum of the type (31) with $m_{1}<m_{2}<\cdots<m_{d} \leq k$ and $\varepsilon_{1}, \ldots, \varepsilon_{d}= \pm 1$. By (30),

$$
l_{i} \in\left(\frac{1}{2} a_{N}^{N}, \frac{3}{2} a_{N}^{N}\right), \quad\left(M_{N-1}<i \leq M_{N}\right) .
$$

Clearly

$$
\prod_{j=1}^{d} \cos 2 \pi \rho_{m_{j}} x=\frac{1}{2^{d-1}} \cos 2 \pi\left(\rho_{m_{d}}+\varepsilon_{d-1} \rho_{m_{d-1}}+\cdots+\varepsilon_{1} \rho_{m_{1}}\right) x
$$

and

$$
\sum_{1 \leq m_{1}<\cdots<m_{d} \leq N} \prod_{j=1}^{d} \cos 2 \pi \rho_{m_{j}} x=\frac{1}{2^{d-1}} \sum_{k=1}^{M_{N}} \cos 2 \pi l_{k} x .
$$

Hence by (29), we have

$$
\varlimsup_{N \rightarrow \infty} \frac{d !}{2^{d-1} \phi_{1}(N)^{d}} \sum_{k=1}^{M_{N}} \cos 2 \pi l_{k} x=1 \quad \text { a.e. }
$$


Put

$$
B_{N}(x)=\max _{M_{N}+1 \leq Q \leq M_{N+1}}\left|\sum_{k=M_{N}+1}^{Q} \cos 2 \pi l_{k} x\right| .
$$

By the Carleson-Hunt inequality [19] we have

$$
\int_{0}^{1} B_{N}^{4}(x) d x \leq C \int_{0}^{1}\left(\sum_{k=M_{N}+1}^{M_{N+1}} \cos 2 \pi l_{k} x\right)^{4} d x
$$

where $C$ is an absolute constant. Put

$$
C_{N}(x)=\sum_{m_{1}, \ldots, m_{d-1} \leq N-1: m_{i} \neq m_{j}(i \neq j)} \prod_{j=1}^{d-1} \cos 2 \pi \rho_{m_{j}} x .
$$

By

$$
\begin{aligned}
\sum_{k=M_{N}+1}^{M_{N+1}} \cos 2 \pi l_{k} x & =2^{d-1} \sum_{m_{1}<\cdots<m_{d-1}<m_{d}=N} \prod_{j=1}^{d} \cos 2 \pi \rho_{m_{j}} x \\
& =\frac{2^{d-1}}{d !} C_{N}(x) \cos 2 \pi N x
\end{aligned}
$$

we have

$$
\int_{0}^{1} B_{N}^{4}(x) d x \leq C\left(\frac{2^{d-1}}{d !}\right)^{4} \int_{0}^{1} C_{N}^{4}(x)
$$

As before, by the inclusion-exclusion principle, we see that $\left|C_{N}(x)\right|$ can be bounded from above by a linear combination of

$$
\left|\prod_{j=1}^{\beta} \sum_{k=1}^{N-1} \cos ^{\alpha_{j}} 2 \pi \rho_{k} x\right| \quad\left(\alpha_{1}+\cdots \alpha_{\beta}=d-1, \alpha_{j} \geq 1\right) .
$$

Put $S=\sum_{j=1}^{\beta} \alpha_{j} \mathbf{1}\left(\alpha_{j}>1\right)$ and $T=\sum_{j=1}^{\beta} \mathbf{1}\left(\alpha_{j}=1\right)$. $S+T=d-1$ is clear. For $\alpha \geq 2$, we bound $\left|\sum_{k=1}^{N-1} \cos ^{\alpha_{j}} 2 \pi \rho_{k} x\right| \leq N \leq N^{\alpha / 2}$ to have

$$
\left|\prod_{j=1}^{\beta} \sum_{k=1}^{N-1} \cos ^{\alpha_{j}} 2 \pi \rho_{k} x\right| \leq N^{S / 2}\left|\sum_{k=1}^{N-1} \cos 2 \pi \rho_{k} x\right|^{T} .
$$

By applying Theorem 8.20 of Zygmund [28], we have

$$
\int_{0}^{1}\left(\prod_{j=1}^{\beta} \sum_{k=1}^{N} \cos ^{\alpha_{j}} 2 \pi \rho_{k} x\right)^{4} d x=O\left(N^{2 S} N^{2 T}\right)=O\left(N^{2(d-1)}\right) .
$$

Therefore we have

$$
\int_{0}^{1} B_{N}^{4}(x) d x=O\left(N^{2(d-1)}\right) \text { and } \sum_{N=1}^{\infty} \int_{0}^{1}\left(\frac{B_{N}(x)}{N^{d / 2}}\right)^{4} d x<\infty .
$$


By applying the Beppo-Levi Theorem we have $B_{N}=o\left(N^{d / 2}\right)$ a.e. By noting $M_{N} \sim N^{d} 2^{d-1} / d$ ! and combining with (33), we have

$$
\varlimsup_{N \rightarrow \infty} \frac{1}{\sqrt{\left(2^{d-1} / d !\right) N(\log \log N)^{d}}} \sum_{i=1}^{N} \cos 2 \pi l_{i} x=1 \quad \text { a.e. }
$$

Put

$$
n_{i}= \begin{cases}l_{i} & \text { if } i \leq M_{k_{0}} \\ l_{M_{k_{0}}}+\left(i-M_{k_{0}}\right) & \text { if } M_{k_{0}}<i<L_{k_{0}} \\ l_{M_{k}+i+1-L_{k}} & \text { if } L_{k} \leq i<L_{k}^{+} \\ n_{L_{k}^{+}-1}+\left(i+1-L_{k}^{+}\right) & \text {if } L_{k}^{+} \leq i<L_{k+1}\left(k \geq k_{0}\right)\end{cases}
$$

We can verify that $\left\{n_{k}\right\}$ is strictly increasing. Actually by (32) and (26), we see

$$
n_{L_{k_{0}}}=l_{M_{k_{0}}+1}>\gamma_{k_{0}+1}^{+} \geq \gamma_{k_{0}+1}^{-}>l_{M_{k_{0}}}+\left(L_{k_{0}}-1-M_{k_{0}}\right)=n_{L_{k_{0}}-1},
$$

and by (27) we see for $k \geq k_{0}$,

$$
n_{L_{k+1}}=l_{M_{k+1}+1}>\gamma_{k+2}^{+} \geq \gamma_{k+2}^{-}>l_{M_{k+1}}+\left(L_{k+1}-L_{k}^{+}\right)=n_{L_{k+1}-1} .
$$

Put $E=\left[1, M_{k_{0}}\right] \cup \bigcup_{k=k_{0}}^{\infty}\left[L_{k}, L_{k}^{+}\right), F=\mathbf{N} \backslash E, E_{N}=E \cap[1, N]$, $F_{N}=F \cap[1, N]$, and $\eta_{N}={ }^{\#} E_{N}$. By $\eta_{L_{k}}=M_{k}+1$, we have $\Psi^{2}\left(L_{k}\right) \sim$ $\left(2^{d-1} / d !\right) \eta_{L_{k}}\left(\log \log \eta_{L_{k}}\right)^{d}$. By $\Psi^{2}\left(L_{k+1}\right) \sim \Psi^{2}\left(L_{k}\right)$, we have

$$
\Psi^{2}(N) \sim\left(2^{d-1} / d !\right) \eta_{N}\left(\log \log \eta_{N}\right)^{d}
$$

By (34), we see that

$$
\varlimsup_{N \rightarrow \infty} \frac{1}{A_{N}} \sum_{i \in E_{N}} \cos 2 \pi n_{i} x=1 \quad \text { a.e. }
$$

holds for $A_{N}=\sqrt{\left(2^{d-1} / d !\right) \eta_{N} \log \log \eta_{N}}$, and by (35) we see that it holds for $A_{N}=\Psi(N)$.

If $N \in\left[L_{k-1}^{+}, L_{k}\right)$, we have $\left|\sum_{i=L_{k-1}^{+}}^{N} \cos 2 \pi n_{i} x\right| \leq 2 /|\sin \pi x|$. By $\Psi^{2}(N) \sim \eta_{L_{k}} \log \log \eta_{L_{k}} \sim\left(2^{d-1} / d !\right) k^{d} \log \log k$, we can see that

$$
\max _{N \in\left[L_{k-1}^{+}, L_{k}\right)}\left|\sum_{i \in F_{N}} \cos 2 \pi n_{i} x\right| \leq \frac{2 k}{|\sin \pi x|}=o(\Psi(N)) \quad \text { a.e. }
$$

Hence we can verify (9). 


\section{REFERENCES}

[1] C. Aistleitner, On the law of the iterated logarithm for the discrepancy of lacunary sequences, Trans. Amer. Math. Soc., 362 (2010), 5967-5982.

[2] C. Aistleitner \& K. Fukuyama, On the law of the iterated logarithm for trigonometric series with bounded gaps, Probab. Theory Related Fields, 154 (2012) 607-620.

[3] C. Aistleitner \& K. Fukuyama, Extremal discrepancy behaviour of lacunary sequences, Monatsh. Math., 177 (2015) 167-184.

[4] C. Aistleitner \& K. Fukuyama, On the law of the iterated logarithm for trigonometric series with bounded gaps II, J. Théor. des Nombres Bordeaux, (to appear).

[5] C. Aistleitner, K. Fukuyama \& Y. Furuya, Optimal bound for the discrepancies of lacunary sequences, Acta Arith., 158 (2013) 229-243.

[6] C. Aistleitner \& G. Larcher, On sequences with prescribed metric discrepancy behavior, Monatsh. Math., (to appear), DOI: 10.1007/s00605-015-0860-2

[7] C. Aistleitner \& G. Larcher, Metric results on the discrepancy of sequence $\left\{a_{n} \alpha\right\}_{n \geq 1}$ modulo one for integer sequence $\left\{a_{n}\right\}$ of polynomial growth, Mathematika, 62 (2016) 478-491.

[8] R. Baker, Metric number theory and the large sieve, J. London Math. Soc. 24 (1981), 34-40.

[9] I. Berkes \& W. Philipp, The size of trigonometric and Walsh series and uniform distribution mod 1, J. London Math. Soc. (2), 50 (1994) 454-464.

[10] P. Bohl, Über ein in der Theorie der säkulären Störungen vorkommendes Problem, J. Reine Angew. Math., 135 (1909), 189-283.

[11] P. Erdős \& S. Gál I, On the law of the iterated logarithm I, II, Nederl. Akad. Wetensch. Proc. Ser. A 58 (Indag. Math. 17) (1955) 65-76, 77-84.

[12] K. Fukuyama, The law of the iterated logarithm for discrepancies of $\left\{\theta^{n} x\right\}$. Acta Math. Hungar., 118 (2008), 155-170.

[13] K. Fukuyama, A law of the iterated logarithm for discrepancies: non-constant limsup, Monatsh. Math., 160 (2010), 143-149.

[14] K. Fukuyama, A central limit theorem and a metric discrepancy result for sequence with bounded gaps, Dependence in probability, analysis and number theory, A volume in memory of Walter Philipp, Eds. I. Berkes, R. Bradley, H. Dehling, M. Peligrad, and R. Tichy, Kendrick press, 2010, pp. 233-246.

[15] K. Fukuyama, Metric discrepancy results for alternating geometric progressions, Monatsh. Math., 171 (2013) 33-63.

[16] K. Fukuyama, A metric discrepancy result for the sequence of powers of minus two, Indag. Math. (NS), 25 (2014) 487-504.

[17] K. Fukuyama \& M. Yamashita, Metric discrepancy results for geometric progressions with large ratios, Monatsh. Math., (to appear), DOI: 10.1007/s00605015-0791-y.

[18] K. Fukuyama, Y. Mitsuhata, Bounded law of the iterated logarithm for discrepancies of permutations of lacunary sequences, Summer School on the Theory of Uniform Distribution, RIMS Kôkyûroku Bessatsu, B29 (2012) 65-88.

[19] R. A. Hunt, On the convergence of Fourier series, Orthogonal Expansions and their Continuous Analogues, (Proc. Conf. Edwardsville, Ill.,1967) 235-255. Southern Illinois Univ. Press, Carbondale, Ill. 1968. 
[20] A. Khintchine, Einige Sätze über Kettenbrüche, mit Anwendungen auf die Theorie der Diophantischen Approximationen, Math. Ann. 92, (1924) 115-125

[21] A. Kolmogoroff, Über das Gesetz des iterierten Logarithmus, Math. Ann. 101, (1929) 126-135

[22] L. Kuipers, H. Niederreiter, Uniform distribution of sequences. Wiley, 1974.

[23] W. Philipp, Limit theorems for lacunary series and uniform distribution mod 1, Acta Arith. 26, (1975) 241-251

[24] W. Sierpiński, Sur la valuer asymptotique d'une certaine somme, Bull. Int. Acad. Polon. Sci. (Cracovie) A, 1910 (1910), 9-11.

[25] S. Takahashi, An asymptotic property of a gap sequence, Proc. Japan Acad., 38 (1962), 101-104.

[26] H. Weyl, Über die Gibbssche Erscheinung und verwandte Konvergenzphänomene, Rend. Circ. Mat. Palermo, 30 (1910) 377-407.

[27] H. Weyl, Über die Gleichverteilung von Zahlen modulo Eins, Math. Ann., 77 (1916), 313-352.

[28] A. Zygmund, Trigonometric series, Vol I., Cambridge University Press, 1959.

Institute of Statistics, Graz University of Technology, KopernikusGasse 24/III, 8010 Graz, Austria

E-mail address: berkes@tugraz.at

Department of Mathematics, Kobe University, Rokko Kobe 657-8501 JAPAN

E-mail address: fukuyama@math.kobe-u.ac.jp

Department of Mathematics, Kobe University, Rokko Kobe 657-8501 JAPAN

E-mail address: tnishi@math.kobe-u.ac.jp 